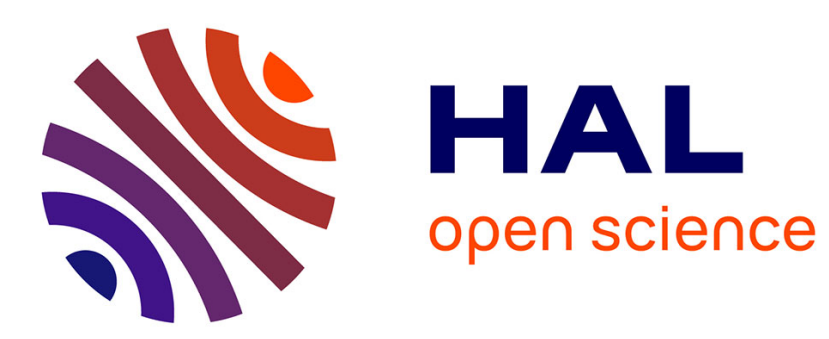

\title{
Closed-Form Expression of the Fourier Ring-Correlation for Single-Molecule Localization Microscopy
} Thanh-An Pham, Emmanuel Soubies, Daniel Sage, Michael Unser

\section{To cite this version:}

Thanh-An Pham, Emmanuel Soubies, Daniel Sage, Michael Unser. Closed-Form Expression of the Fourier Ring-Correlation for Single-Molecule Localization Microscopy. ISBI 2019 - IEEE International Symposium on Biomedical Imaging, Apr 2019, Venise, Italy. hal-02048083

\section{HAL Id: hal-02048083 https://hal.science/hal-02048083}

Submitted on 25 Feb 2019

HAL is a multi-disciplinary open access archive for the deposit and dissemination of scientific research documents, whether they are published or not. The documents may come from teaching and research institutions in France or abroad, or from public or private research centers.
L'archive ouverte pluridisciplinaire HAL, est destinée au dépôt et à la diffusion de documents scientifiques de niveau recherche, publiés ou non, émanant des établissements d'enseignement et de recherche français ou étrangers, des laboratoires publics ou privés. 


\title{
CLOSED-FORM EXPRESSION OF THE FOURIER RING-CORRELATION FOR SINGLE-MOLECULE LOCALIZATION MICROSCOPY
}

\author{
Thanh-an Pham, Emmanuel Soubies, Daniel Sage, and Michael Unser
}

Biomedical Imaging Group, Ecole polytechnique fédérale de Lausanne (EPFL), 1015 Lausanne, Switzerland

\begin{abstract}
Single-molecule localization microscopy (SMLM) is a popular microscopic technique that achieves super resolution imaging by localizing individual blinking molecules in thousands of frames. Therefore, the reconstructed high-resolution image is a combination of millions of point sources. This particular computational reconstruction leads to the question of the estimation of the image resolution. Fourier-ring correlation (FRC) is the standard tool for assessing the resolution. It has been proposed for SMLM by computing a discrete correlation in the Fourier domain. In this work, we derive a closed-form expression to compute the continuous FRC. Our implementation provides an exact FRC and an alternative to compute a parameter-free FRC. In addition, it gives insights on the discrepancy of the discrete FRC and yields a rule to select its parameters such as the spatial sampling step or the width of the kernel used as density estimator.
\end{abstract}

Index Terms-Single-molecule localization microscopy, Fourierring correlation, image resolution.

\section{INTRODUCTION}

Single-molecule localization microscopy (SMLM) is a superresolution microscopy technique capable of imaging objects of the order of tens of nanometers. This modality, also known as PALM or STORM, has become a standard tool in cell biology [1]. This computational imaging combines an optical setup and a localization algorithm to render an image reconstructed from a set of estimated positions. The rendering process usually involves a kernel that determines the contribution of each position to the reconstructed image [2, 3]. Since the emergence of SMLM, the determination of image resolution has become a primordial matter as the reconstructed image is a combination of optics and numerics.

The Fourier-ring correlation (FRC) [4] or, equivalently, the spectral signal-to-noise ratio (SSNR) [5] is a standard tool for resolution assessment in electron microscopy. It has been recently extended to SMLM [6, 7] and quickly adopted by the community as a standard indicator of resolution. The FRC is computed from discretized rendered images. It therefore depends on experimental parameters such as the pixel size or the choice of density estimator.

In this work, we take into consideration the specificity of SMLM to derive a closed-form expression of the FRC. It is noteworthy to mention that, in the current development of localization microscopy, doing post-analysis directly on the coordinates itself and bypassing

This research was supported by the European Research Council (ERC) under the European Union's Horizon 2020 research and innovation programme, Grant Agreement no. 692726 "GlobalBioIm: Global integrative framework for computational bio-imaging." Funding: European Research Council (ERC) (692726). the image binning step is popular $[8,9]$. We first proceed by introducing the mathematical definition of the FRC (Section 2) and its conventional (discrete) computation, for which we derive an error bound (Section 3). We then derive the closed-form expression in the continuous domain and we address specific points of implementation (Section 4). Finally, we assess the difference between a FRC computed in a conventional way (discretized) and the proposed closed-form expression with a dataset from the SMLM challenge [10] (Section 5).

\section{NOTATIONS AND DEFINITIONS}

Definition 2.1 (SMLM image rendering). Given the set $\mathcal{P}=\left\{\mathbf{p}_{n} \in\right.$ $\left.\mathbb{R}^{2}\right\}_{n=1}^{N}$ that contains the positions of $N \in \mathbb{N}$ molecules, we define the image $f_{\mathcal{P}} \in L_{2}\left(\mathbb{R}^{2}\right)$ by

$$
f_{\mathcal{P}}(\mathbf{x})=\sum_{n=1}^{N}\left(\Phi_{n} * \delta\left(\cdot-\mathbf{p}_{n}\right)\right)(\mathbf{x}), \mathbf{x} \in \mathbb{R}^{2},
$$

where the elements of $\left\{\Phi_{n} \in L_{2}\left(\mathbb{R}^{2}\right)\right\}_{n=1}^{N}$ are called the rendering kernels.

Assumption 2.2. The rendering kernels are compactly supported radial functions (i.e., there exist compactly supported $1 D$ functions $\phi_{n} \in L_{2}\left(\mathbb{R}_{\geq 0}\right)$ such that $\left.\Phi_{n}(\mathbf{x})=\phi_{n}(\|\mathbf{x}\|)\right)$. Hence, their Fourier transform is also radial, so that we write $\hat{\Phi}_{n}(\boldsymbol{\omega})=\hat{\phi}_{n}(\|\boldsymbol{\omega}\|)$.

From Definition 2.1, each molecule can be rendered using a different kernel. For instance, one can use truncated Gaussian kernels whose variances are related to the number of detected photons $[11,12,13,14,15,16]$. From Assumption 2.2 and Definition 2.1, we readily deduce that the rendered image $f_{\mathcal{P}}$ is compactly supported. Using the translation property of the Fourier transform, we also get that

$$
\hat{f}_{\mathcal{P}}(\boldsymbol{\omega})=\sum_{n=1}^{N} \hat{\Phi}_{n}(\boldsymbol{\omega}) \mathrm{e}^{-\mathrm{i} \mathbf{p}_{n}^{T} \boldsymbol{\omega}} .
$$

Definition 2.3 (Fourier-ring correlation (FRC) [6, 7]). Let $f \in$ $L_{2}\left(\mathbb{R}^{2}\right)$ and $g \in L_{2}\left(\mathbb{R}^{2}\right)$. Then, the FRC between the images $f$ and $g$ is defined $\forall \rho>0$ as

$$
\operatorname{FRC}_{\{f, g\}}(\rho)=\frac{\langle\hat{f}, \hat{g}\rangle_{\mathcal{C}_{\rho}}}{\sqrt{\langle\hat{f}, \hat{f}\rangle_{\mathcal{C}_{\rho}}\langle\hat{g}, \hat{g}\rangle_{\mathcal{C}_{\rho}}}},
$$

where $\mathcal{C}_{\rho}:=\left\{\boldsymbol{\omega} \in \mathbb{R}^{2}:\|\boldsymbol{\omega}\|_{2}=\rho\right\}$ is a circle of radius $\rho>0$ and

$$
\langle\hat{f}, \hat{g}\rangle_{\mathcal{C}_{\rho}}:=\oint_{\mathcal{C}_{\rho}} \hat{f}^{\star}(\boldsymbol{\omega}) \hat{g}(\boldsymbol{\omega}) \mathrm{d} \boldsymbol{\omega} .
$$




\section{CONVENTIONAL FRC COMPUTATION IN SMLM}

To compute the FRC, the standard practice is to sample the continuously rendered images $f_{\mathcal{P}} \in L_{2}\left(\mathbb{R}^{2}\right)$ and $f_{\mathcal{Q}} \in L_{2}\left(\mathbb{R}^{2}\right)$ as

$$
\begin{aligned}
& \mathbf{f}_{\mathcal{P}} \in \mathbb{R}^{K} \text { such that }\left[\mathbf{f}_{\mathcal{P}}\right]_{k}=f_{\mathcal{P}}\left(\mathbf{x}_{k}\right) \\
& \mathbf{f}_{\mathcal{Q}} \in \mathbb{R}^{K} \text { such that }\left[\mathbf{f}_{\mathcal{Q}}\right]_{k}=f_{\mathcal{Q}}\left(\mathbf{x}_{k}\right),
\end{aligned}
$$

where $\left\{\mathbf{x}_{k} \in \mathbb{R}^{2}\right\}_{k=1}^{K}$ is a set of sampling points on a uniform Cartesian grid. We denote by $s>0$ the spatial sampling step. The size of the grid is chosen such that the (compact) support of $f_{\mathcal{P}}\left(f_{\mathcal{Q}}\right.$, respectively) is fully contained within the discrete image $\mathbf{f}_{\mathcal{P}}\left(\mathbf{f}_{\mathcal{Q}}\right.$, respectively). Then, the FRC is computed from the discrete Fourier transform (DFT) of $\mathbf{f}_{\mathcal{P}}$ and $\mathbf{f}_{\mathcal{Q}}$ (which are denoted $\hat{\mathbf{f}}_{\mathcal{P}}$ and $\hat{\mathbf{f}}_{\mathcal{Q}}$ ), using numerical integration and interpolation. Henceforth, we shall refer to this approach as discrete FRC. From the Poisson summation formula, we have that

$$
\left[\hat{\mathbf{f}}_{\mathcal{P}}\right]_{k}=\sum_{\mathbf{m} \in \mathbb{Z}^{2}} \hat{f}_{\mathcal{P}}\left(\boldsymbol{\omega}_{k}+2 \pi s^{-1} \mathbf{m}\right)
$$

where $\boldsymbol{\omega}_{k}$ is the pulsation that corresponds to $\left[\hat{\mathbf{f}}_{\mathcal{P}}\right]_{k}$. As a result, the sampling procedure yields the error

$$
\begin{aligned}
\left|\left[\hat{\mathbf{f}}_{\mathcal{P}}\right]_{k}-\hat{f}_{\mathcal{P}}\left(\boldsymbol{\omega}_{k}\right)\right| \leq \sum_{\mathbf{m} \in \mathbb{Z}^{2} \backslash\{\mathbf{0}\}}\left|\hat{f}_{\mathcal{P}}\left(\boldsymbol{\omega}_{k}+2 \pi s^{-1} \mathbf{m}\right)\right| \\
\leq \sum_{\mathbf{m} \in \mathbb{Z}^{2} \backslash\{\mathbf{0}\}} \sum_{n=1}^{N}\left|\hat{\Phi}_{n}\left(\boldsymbol{\omega}_{k}+2 \pi s^{-1} \mathbf{m}\right)\right| .
\end{aligned}
$$

Because the kernels $\Phi_{n}$ are compactly supported (Assumption 2.2), their Fourier transforms $\hat{\Phi}_{n}$ are not. Hence, the accuracy of the discrete FRC is related to the sampling step $s$ as well as the decay of $\left|\hat{\Phi}_{n}\right|$. They have to be tuned so as to minimize the aliasing (i.e., minimize the bound in (8)). In particular, the sampling step must be sufficiently small and $\left|\hat{\Phi}_{n}\right|$ must decrease fast enough. This will be further discussed along with the numerical experiments (Section 5).

\section{CLOSED-FORM EXPRESSION OF THE FRC IN CONTINUOUS DOMAIN}

\subsection{Main Result}

We present our main result in Proposition 4.1 where we derive a closed-form expression of the FRC in the continuous domain. Henceforth, we shall refer to our approach as closed-form FRC.

Proposition 4.1. Let $f_{\mathcal{P}} \in L_{2}\left(\mathbb{R}^{2}\right)$ and $f_{\mathcal{Q}} \in L_{2}\left(\mathbb{R}^{2}\right)$ be the rendered images of $\mathcal{P}=\left\{\mathbf{p}_{n} \in \mathbb{R}^{2}\right\}_{n=1}^{N}$ and $\mathcal{Q}=\left\{\mathbf{q}_{m} \in \mathbb{R}^{2}\right\}_{m=1}^{M}$, respectively. Then, the FRC is given by (3) with

$$
\left\langle\hat{f}_{\mathcal{P}}, \hat{f}_{\mathcal{Q}}\right\rangle_{\mathcal{C}_{\rho}}=\sum_{n=1}^{N} \sum_{m=1}^{M}\left(\hat{\phi}_{n} \hat{\phi}_{m}^{\star}\right)(\rho) J_{0}\left(\left\|\mathbf{p}_{n}-\mathbf{q}_{m}\right\|_{2} \rho\right),
$$

where $J_{0}$ is the zero-order Bessel function of the first kind. The kernels $\hat{\phi}_{n}$ follow the conditions in Assumption 2.2.

Proof. Injecting (2) into $\left\langle\hat{f}_{\mathcal{P}}, \hat{f}_{\mathcal{Q}}\right\rangle_{\mathcal{C}_{\rho}}$, we obtain that

$$
\left\langle\hat{f}_{\mathcal{P}}, \hat{f}_{\mathcal{Q}}\right\rangle_{\mathcal{C}_{\rho}}=\oint_{\mathcal{C}_{\rho}} \sum_{n, m=1}^{N, M}\left(\hat{\Phi}_{n} \hat{\Phi}_{m}^{\star}\right)(\boldsymbol{\omega}) \mathrm{e}^{-\mathrm{i}\left(\mathbf{p}_{n}-\mathbf{q}_{m}\right)^{T} \boldsymbol{\omega}} \mathrm{d} \boldsymbol{\omega} .
$$

By definition, we have that $\forall \boldsymbol{\omega} \in \mathcal{C}_{\rho},\|\boldsymbol{\omega}\|_{2}=\rho$. Combining that fact with Assumption 2.2 and the linearity of the integral comes to

$$
\left\langle\hat{f}_{\mathcal{P}}, \hat{f}_{\mathcal{Q}}\right\rangle_{\mathcal{C}_{\rho}}=\sum_{n, m=1}^{N, M}\left(\hat{\phi}_{n} \hat{\phi}_{m}^{\star}\right)(\rho) \oint_{\mathcal{C}_{\rho}} \mathrm{e}^{-\mathrm{i} \mathbf{r}_{n m}^{T} \boldsymbol{\omega}} \mathrm{d} \boldsymbol{\omega},
$$

where $\mathbf{r}_{n m}=\left(\mathbf{p}_{n}-\mathbf{q}_{m}\right)$. By setting $\mathbf{r}_{n m}=r_{n m}\left(\cos \left(\theta_{n m}\right)\right.$, $\left.\sin \left(\theta_{n m}\right)\right)$ and $\boldsymbol{\omega}=\omega(\cos (\theta), \sin (\theta))$ (polar coordinates), we have that

$$
\begin{aligned}
\mathbf{r}_{n m}^{T} \boldsymbol{\omega} & =r_{n m} \omega\left(\cos \left(\theta_{n m}\right) \cos (\theta)+\sin \left(\theta_{n m}\right) \sin (\theta)\right) \\
& =r_{n m} \omega \cos \left(\theta-\theta_{n m}\right) .
\end{aligned}
$$

The integral in (11) becomes

$$
\begin{aligned}
\oint_{\mathcal{C}_{\rho}} \mathrm{e}^{-\mathrm{i} \mathbf{r}_{n m}^{T} \boldsymbol{\omega}} \mathrm{d} \boldsymbol{\omega} & =\int_{-\pi}^{\pi} e^{-\mathrm{i} r_{n m} \rho \cos \left(\theta-\theta_{n m}\right)} \mathrm{d} \theta \\
& =\int_{-\pi}^{\pi} \mathrm{e}^{-\mathrm{i} r_{n m} \rho \sin (\theta)} \mathrm{d} \theta \\
& =2 \pi J_{0}\left(r_{n m} \rho\right) .
\end{aligned}
$$

The second equality comes from the fact that $\cos \left(\theta-\theta_{n m}\right)=$ $\sin \left(\theta-\theta_{n m}+\pi / 2\right)$ and that $\sin$ is a $2 \pi$-periodic function. By inserting (13) into (11), we obtain (9).

This continuous-domain expresssion allows for computing the FRC over circles, while in the discrete case it must be computed over annuli (circles with a certain "width"). A direct consequence of Proposition 4.1 is that, when the same rendering kernel $\Phi$ is used for all the molecules, the FRC does not depend anymore on $\Phi$.

Corollary 4.2. Let $\Phi \in L_{2}\left(\mathbb{R}^{2}\right)$ be a rendering kernel that fulfills Assumption 2.2 and let $\Phi_{n}=\Phi_{m}=\Phi$ for all $n \in[1 \ldots N], m \in$ $[1 \ldots M]$. Then, the FRC does not depend on $\Phi$.

Proof. Because $\Phi$ fulfills Assumption 2.2, we have that $\hat{\Phi}=\hat{\phi}(\|\cdot\|)$ for a given $\hat{\phi} \in L_{2}\left(\mathbb{R}_{\geq 0}\right)$. Hence, (9) becomes

$$
\left\langle\hat{f}_{\mathcal{P}}, \hat{f}_{\mathcal{Q}}\right\rangle_{\mathcal{C}_{\rho}}=|\hat{\phi}(\rho)|^{2} \sum_{n, m=1}^{N, M} J_{0}\left(\left\|\mathbf{p}_{n}-\mathbf{q}_{m}\right\|_{2} \rho\right) .
$$

The term $|\hat{\phi}(\rho)|^{2}$ is ultimately cancelled by the denominator in (3), which completes the proof.

\subsection{Practical Implementation}

To compute the closed-form FRC, we must compute three instances of an expression of the same type as (9). Each instance requires the calculation of the Euclidean distance between each point of one set of positions with each point of another set of positions (or itself). This yields a computational cost of $O\left(N^{2}+M N+M^{2}\right)$ for one value of $\rho$. For illustration, we report running times with and without GPU in Table 1.

\section{NUMERICAL EXPERIMENTS}

\subsection{Continuous vs. Discrete FRC}

In this section, we compare the conventional FRC computation (Section 3 ) to the proposed closed-form expression (Section 4). 

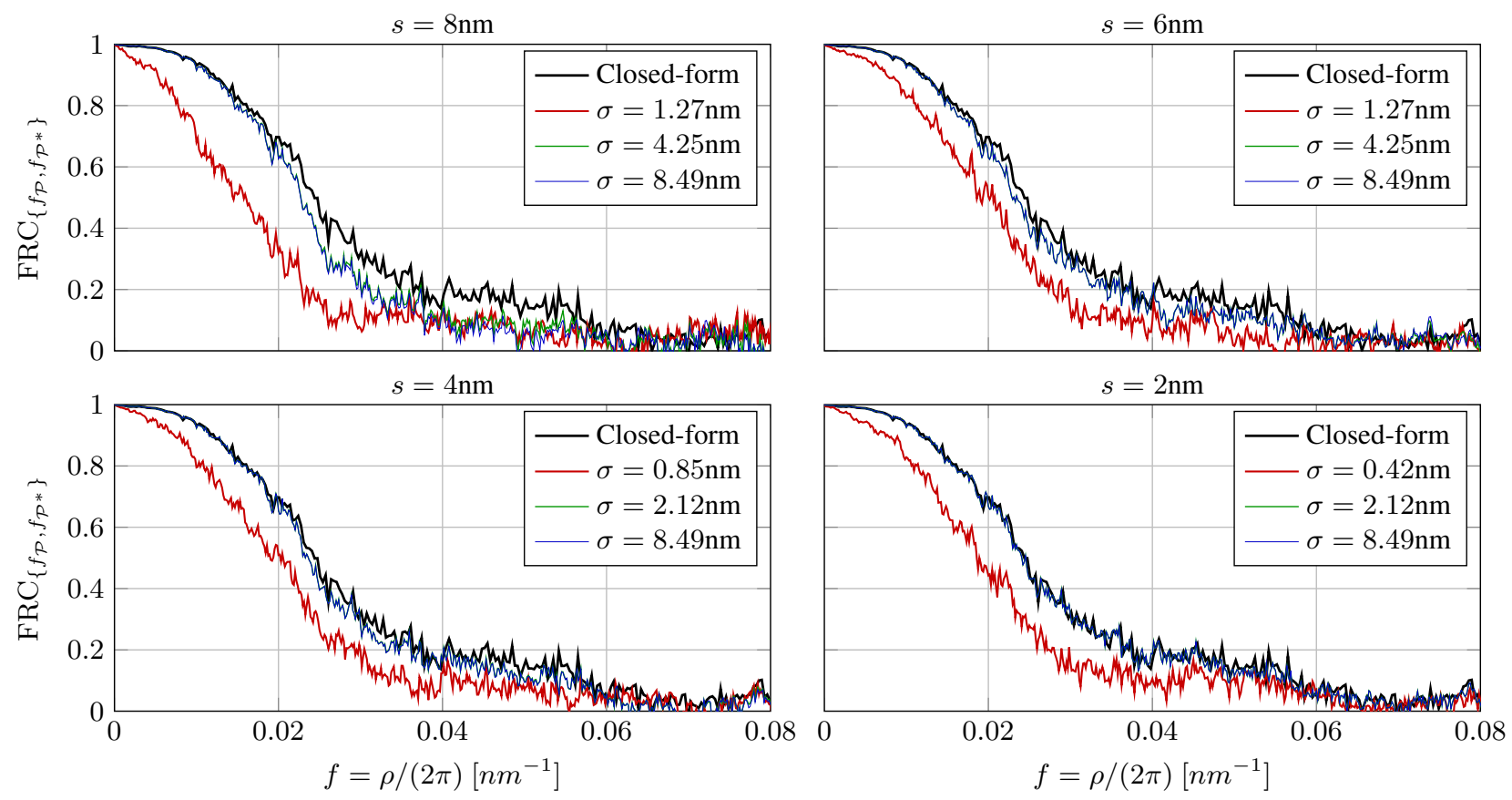

Fig. 1: Comparison of the closed-form FRC curve with discrete FRC curves obtained using different sampling steps $s$ and rendering kernels $\Phi_{\sigma}$. The discrete FRC curves reach the closed-form FRC curve when the width of the kernel and the sampling step are appropriately set (e.g., $s=2 \mathrm{~nm}$ and $\sigma=8.49 \mathrm{~nm})$.

Table 1: Running time to compute the closed-form FRC. The two sets have $M=3647$ and $N=5514$ positions respectively. Three hundred samples of the closed-form FRC were computed. As a reference, the running times for the discrete FRC (three hundred points, image size $3200 \times 3200$ ) was 0.3 s on CPU.

\begin{tabular}{ll}
\hline \hline $\mathrm{CPU}$ & $283 \mathrm{~s}$ \\
$\mathrm{GPU}$ & $6.6 \mathrm{~s}$ \\
\hline \hline
\end{tabular}

We consider the MT1.N1.LD dataset of the 3D SMLM challenge ${ }^{1}$ and we denote by $\mathcal{P}^{*}$ the set containing the positions of the ground-truth molecules. The second set, $\mathcal{P}$, contains the positions of the molecules which were localized by one of the participants [17] for the MT1.N1.LD dataset. We use a unique rendering kernel for all the molecules and define it as the truncated Gaussian

$$
\Phi_{\sigma}(\mathbf{x})=\phi_{\sigma}(\|\mathbf{x}\|)
$$

where, for $\sigma>0$,

$$
\phi_{\sigma}(x)= \begin{cases}\frac{1}{\sigma \sqrt{2 \pi}} \mathrm{e}^{-\frac{x^{2}}{2 \sigma^{2}}} & |x|<5 \sigma \\ 0, & \text { otherwise. }\end{cases}
$$

We computed some discrete FRC with different sampling steps $(s=2,4,6,8 \mathrm{~nm})$ and different widths of the kernel $(\sigma=$ $\{0.42,0.85,1.27,2.12,4.25,8.49\} \mathrm{nm})$. These values were computed from arbitrary chosen full width at half maximum $(\mathrm{FWHM}=$

\footnotetext{
${ }^{1}$ Single-molecule localization microscopy software benchmarking, http://bigwww.epfl.ch/smlm/challenge2016/, accessed Octobre 16, 2018.
}

$2 \sqrt{2 \log 2} \sigma=\{1,2,3,5,10,20\})$. In Figure 1, we show some of these curves for the sake of visibility. We observe that the conventional FRC curve reaches the closed-form FRC for some values of the sampling step of the discrete rendered image, as well as the parameters of the rendering kernels.

\subsection{Quantitative Discrepancy of the Discrete FRC}

In Figure 2, we compute the relative discrepancy between the discrete FRC and the closed-form FRC defined as

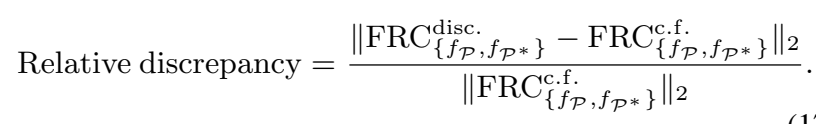

We observe that a smaller sampling step yields a better approximation, as expected. In addition, for each sampling step, there exists a range of widths of kernel for which the discrepancy is minimized. Finally, we can relate these observations to the error bound derived in Section 3. Indeed, when the step size is small enough, small values of $\sigma$ are sufficient to minimize the error to the closed-form FRC. However, if one chooses a larger step size, the parameter $\sigma$ has to be larger as well in order to reduce the aliasing effect and minimize the error.

\section{CONCLUSION}

In this work, we obtained a closed-form expression to compute the Fourier-ring correlation (FRC) in the continuous domain for SMLM. Our method allows us to compute a parameter-free FRC. We showed that the conventional FRC computation reaches the closed-form FRC when the sampling parameters are set appropriately. Moreover, our approach could allow for an accurate computation of the local 


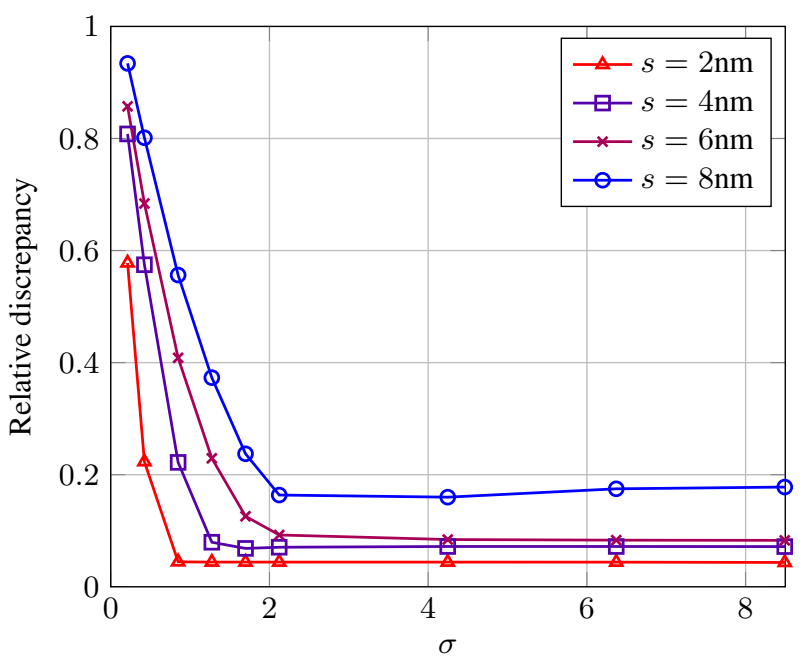

Fig. 2: Relative discrepancy between the discrete and the closedform FRC for different values of kernel width $\sigma$. For any sampling step, there exists a range of parameters for the discrete FRC which minimizes the discrepancy.

FRC [18]. Future works should consider other image-based metrics such as SNR.

\section{REFERENCES}

[1] M. Sauer, "Localization microscopy coming of age: From concepts to biological impact," Journal of Cell Science, vol. 126, no. 16, pp. 3505-3513, 2013.

[2] T. Pengo, S. J. Holden, and S. Manley, "PALMsiever: A tool to turn raw data into results for single-molecule localization microscopy," Bioinformatics, vol. 31, no. 5, pp. 797-798, 2014.

[3] D. Baddeley, M. B. Cannell, and C. Soeller, "Visualization of localization microscopy data," Microscopy and Microanalysis, vol. 16, no. 1, pp. 64-72, 2010.

[4] W.O. Saxton and W. Baumeister, "The correlation averaging of a regularly arranged bacterial cell envelope protein," Journal of Microscopy, vol. 127, no. 2, pp. 127-138, 1982.

[5] M. Unser, B. L. Trus, and A. C. Steven, "A new resolution criterion based on spectral signal-to-noise ratios," Ultramicroscopy, vol. 23, no. 1, pp. 39-51, 1987.

[6] N. Banterle, K. H. Bui, E. A. Lemke, and M. Beck, "Fourier ring correlation as a resolution criterion for super-resolution microscopy," Journal of Structural Biology, vol. 183, no. 3, pp. 363-367, 2013.

[7] R. P.J. Nieuwenhuizen, K. A. Lidke, M. Bates, D. L. Puig, D. Grünwald, S. Stallinga, and B. Rieger, "Measuring image resolution in optical nanoscopy," Nature Methods, vol. 10, no. 6, pp. 557, 2013.

[8] Hamidreza Heydarian, Florian Schueder, Maximilian T Strauss, Ben Van Werkhoven, Mohamadreza Fazel, Keith A Lidke, Ralf Jungmann, Sjoerd Stallinga, and Bernd Rieger, "Template-free $2 \mathrm{~d}$ particle fusion in localization microscopy," Nature methods, vol. 15, no. 10, pp. 781, 2018.
[9] Joerg Schnitzbauer, Yina Wang, Shijie Zhao, Matthew Bakalar, Tulip Nuwal, Baohui Chen, and Bo Huang, "Correlation analysis framework for localization-based superresolution microscopy," Proceedings of the National Academy of Sciences, vol. 115, no. 13, pp. 3219-3224, 2018.

[10] D. Sage, H. Kirshner, T. Pengo, N. Stuurman, J. Min, S. Manley, and M. Unser, "Quantitative evaluation of software packages for single-molecule localization microscopy," Nature Methods, vol. 12, no. 8, pp. 717, 2015.

[11] R. E. Thompson, D. R. Larson, and W. W. Webb, "Precise nanometer localization analysis for individual fluorescent probes," Biophysical Journal, vol. 82, no. 5, pp. 2775-2783, 2002.

[12] R. J. Ober, S. Ram, and E. S. Ward, "Localization accuracy in single-molecule microscopy," Biophysical Journal, vol. 86, no. 2, pp. 1185-1200, 2004.

[13] H. Deschout, F. C. Zanacchi, M. Mlodzianoski, A. Diaspro, J. Bewersdorf, S. T. Hess, and K. Braeckmans, "Precisely and accurately localizing single emitters in fluorescence microscopy," Nature Methods, vol. 11, no. 3, pp. 253, 2014.

[14] K. I. Mortensen, L. S. Churchman, J. A. Spudich, and H. Flyvbjerg, "Optimized localization analysis for single-molecule tracking and super-resolution microscopy," Nature Methods, vol. 7, no. 5, pp. 377, 2010.

[15] C. S. Smith, N. Joseph, B. Rieger, and K. A. Lidke, "Fast, single-molecule localization that achieves theoretically minimum uncertainty," Nature Methods, vol. 7, no. 5, pp. 373, 2010.

[16] S. Stallinga and B. Rieger, "The effect of background on localization uncertainty in single emitter imaging," in 9th IEEE International Symposium on Biomedical Imaging (ISBI), Barcelona, Spain, 2-5 May 2012, IEEE, pp. 988-991.

[17] Y. Li, M. Mund, P. Hoess, J. Deschamps, U. Matti, B. Nijmeijer, V. J. Sabinina, J. Ellenberg, I. Schoen, and J. Ries, "Realtime 3D single-molecule localization using experimental point spread functions," Nature Methods, vol. 15, no. 5, pp. 367, 2018.

[18] S. Culley, D. Albrecht, C. Jacobs, P. M. Pereira, C. Leterrier, J. Mercer, and R. Henriques, "Quantitative mapping and minimization of super-resolution optical imaging artifacts," Nature Methods, vol. 15, no. 4, pp. 263, 2018. 